\title{
A FIXED POINT APPROACH TO THE STABILITY OF QUINTIC MAPPINGS IN QUASI $\beta$-NORMED SPACES
}

\author{
HEEJEONG KOH*
}

\begin{abstract}
We investigate the general solution of the following functional equation and the generalized Hyers-Ulam-Rassias stability problem in quasi $\beta$-normed spaces and then the stability by using alternative fixed point method for the following quintic function $f: X \rightarrow Y$ such that

$$
\begin{aligned}
& f(3 x+y)+f(3 x-y)+5[f(x+y)+f(x-y)] \\
& =4[f(2 x+y)+f(2 x-y)]+2 f(3 x)-246 f(x),
\end{aligned}
$$

for all $x, y \in X$.
\end{abstract}

\section{Introduction}

More than a half century ago, Ulam [23] proposed the famous problem concerning the stability of group homomorphisms as follows: Let $G_{1}$ be a group and let $G_{2}$ be a metric group with the metric $d(\cdot, \cdot)$. Given $\varepsilon>0$, does there exist a $\delta>0$ such that if a function $h: G_{1} \rightarrow G_{2}$ satisfies the inequality $d(h(x y), h(x) h(y))<\delta$ for all $x, y \in G_{1}$ then there is a homomorphism $H: G_{1} \rightarrow G_{2}$ with $d(h(x), H(x))<\varepsilon$ for all $x \in G_{1}$ ? In other words, when is it true that a mapping satisfying a functional equation approximately must be close to the solution of the given functional equation ? In 1941, Hyers [9] considered the case of approximately additive mappings under the assumption that $G_{1}$ and $G_{2}$ are Banach spaces.

The famous Hyers stability result that appeared in [9] was generalized by Aoki [1] for the stability of the additive mapping involving a sum of powers of $p$-norms. In 1978, Rassias [16] provided a generalization of Hyers' Theorem for the stability of the linear mapping, which allows the Cauchy difference to be unbounded. He considered a mapping $f: X \rightarrow$

Received July 15, 2013; Accepted September 27, 2013.

2010 Mathematics Subject Classification: Primary 39B52; Secondary 47B47.

Key words and phrases: Hyers-Ulam-Rassias stability, functional equation, quintic mapping, quasi $\beta$-mormed space, alternative fixed point. 
$Y$ satisfying the condition $\|f(x+y)-f(x)-f(y)\| \leq \varepsilon\left(\|x\|^{p}+\|y\|^{p}\right)$ for all $x, y \in X$, where $\varepsilon \geq 0$ and $0 \leq p<1$. This theorem was later extended for all $p \neq 1$ and this result of Rassias lead mathematicians working in stability of functional equations to establish what is known today as Hyers-Ulam-Rassias stability or Cauchy-Rassias stability as well as to introduce new definitions of stability concepts. During the last three decades, several stability problems of a large variety of functional equations have been extensively studied and generalized by a number of authors [2],[5],[12],[6],[7],[10],[17],,[11],[18],[19], and [20]. In particular, Cho and et al. [4] introduced the quintic functional equation

$$
\begin{aligned}
& 2 f(2 x+y)+2 f(2 x-y)+f(x+2 y)+f(x-2 y) \\
& =20[f(x+y)+f(x-y)]+90 f(x) .
\end{aligned}
$$

It is easy to see that $f(x)=x^{5}$ is a solution of (1.1) by virtue of the identity

$2(2 x+y)^{5}+2(2 x-y)^{5}+(x+2 y)^{5}+(x-2 y)^{5}=20\left[(x+y)^{5}+(x-y)^{5}\right]+90 x^{5}$.

For this reason, (1.1) is called a quintic functional equation. Also Xu and et al. [24], Gordji and et al. [8] and Park [14] introduced a quintic mapping and sextic mapping.

We will use the following definition to prove Hyers-Ulam-Rassias stability for a quintic functional equation in the quasi $\beta$-normed space. Let $\beta$ be a real number with $0<\beta \leq 1$ and $\mathbb{K}$ be either $\mathbb{R}$ or $\mathbb{C}$.

Definition 1.1. Let $X$ be a linear space over a field $\mathbb{K}$. A quasi $\beta$-norm $\|\cdot\|$ is a real-valued function on $X$ satisfying the following statements:

(1) $\|x\| \geq 0$ for all $x \in X$ and $\|x\|=0$ if and only if $x=0$.

(2) $\|\lambda x\|=|\lambda|^{\beta} \cdot\|x\|$ for all $\lambda \in \mathbb{K}$ and all $x \in X$.

(3) There is a constant $K \geq 1$ such that $\|x+y\| \leq K(\|x\|+\|y\|)$ for all $x, y \in X$.

The pair $(X,\|\cdot\|)$ is called a quasi $\beta$-normed space if $\|\cdot\|$ is a quasi $\beta$-norm on $X$. The smallest possible $K$ is called the modulus of concavity of $\|\cdot\|$. A quasi $\beta$-Banach space is a complete quasi- $\beta$-normed space.

A quasi $\beta$-norm $\|\cdot\|$ is called a $(\beta, p)$-norm $(0<p \leq 1)$ if $(3)$ takes the form $\|x+y\|^{p} \leq\|x\|^{p}+\|y\|^{p}$ for all $x, y \in X$. In this case, a quasi $\beta$-Banach space is called a $(\beta, p)$-Banach space; see [3], [21] and [15].

In this paper, we deal with the following the functional equation:

$$
\begin{aligned}
& f(3 x+y)+f(3 x-y)+5[f(x+y)+f(x-y)] \\
& =4[f(2 x+y)+f(2 x-y)]+2 f(3 x)-246 f(x),
\end{aligned}
$$


for all $x, y \in X$. We investigate the general solution of the functional equation and generalized Hyers-Ulam-Rassias stability problem in quasi $\beta$-normed spaces and then the stability by using a alternative fixed point method for the function $f: X \rightarrow Y$ satisfying the equation (1.2).

\section{Quintic functional equations}

In this section let $X$ and $Y$ be vector spaces and we investigate the general solution of the functional equation (1.2). Some basic facts on $n$-additive symmetric mappings can be found in [24].

Theorem 2.1. A function $f: X \rightarrow Y$ is a solution of the functional equation (1.2) if and only if $f$ is of the form $f(x)=A^{5}(x)$ for all $x \in X$, where $A^{5}(x)$ is the diagonal of the 5-additive symmetric map $A_{5}: X^{5} \rightarrow$ $Y$.

Proof. Suppose $f$ satisfies the functional equation (1.2). Letting $x=$ $y=0$ in the equation (1.2), we have $12 f(0)=-235 f(0)$, that is, $f(0)=$ 0 . Letting $x=0$ and replacing $y$ by $x$ in the equation (1.2), we have $f(x)=-f(x)$, for all $x \in X$. Hence $f$ is an odd mapping. Putting $y=0$ in the equation (1.2), we get $f(2 x)=32 f(x)$, for all $x \in X$. Hence we have

$$
f\left(2^{n} x\right)=32^{n} f(x),
$$

for all $x \in X$ and $n \in \mathbb{N}$. Note that $f(x)=\frac{1}{32^{n}} f\left(2^{n} x\right)$, for all $x \in X$ and $n \in \mathbb{N}$. Now, letting $x=y$ in the equation (1.2), we have $f(3 x)=$ $243 f(x)$ for all $x \in X$.

On the other hand, we can rewrite the functional equation (1.2) in the form

$$
\begin{aligned}
f(x) & -\frac{1}{240} f(3 x+y)-\frac{1}{240} f(3 x-y)+\frac{1}{60} f(2 x+y)+\frac{1}{60} f(2 x-y) \\
& -\frac{1}{48} f(x+y)-\frac{1}{48} f(x-y)=0,
\end{aligned}
$$

for all $x \in X$. By [24, Theorem 3.5 and Theorem 3.6] $f$ is a general polynomial function of degree at most 5 , that is, $f$ is of the following form

$$
f(x)=A^{5}(x)+A^{4}(x)+A^{3}(x)+A^{2}(x)+A^{1}(x)+A^{0}(x)
$$

for all $x \in X$. Note that $A^{0}(x)=A^{0}$ is an arbitrary element of $Y$ and $A^{i}(x)$ is the diagonal of the $i$-additive symmetric map $A_{i}: X^{i} \rightarrow Y$ for $i=1,2,3,4,5$. Since $f(0)=0$ and $f$ is odd, we have $A^{0}(x)=A^{0}=0$ 
and $A^{4}(x)=A^{2}(x)=0$. It follows that $f(x)=A^{5}(x)+A^{3}(x)+A^{1}(x)$, for all $x \in X$. By (2.1) and $A^{n}(r x)=r^{n} A^{n}(x)$ whenever $x \in X$ and $r \in \mathbb{Q}$, we obtain

$$
\begin{aligned}
& 2^{5} A^{5}(x)+2^{3} A^{3}(x)+2 A^{1}(x) \\
& =f(2 x)=2^{5} f(x)=2^{5} A^{5}(x)+2^{5} A^{3}(x)+2^{5} A^{1}(x),
\end{aligned}
$$

for all $x \in X$. Then $A^{1}(x)=-\frac{4}{5} A^{3}(x)$, for all $x \in X$. Hence $A^{3}(x)=$ $A^{1}(x)=0$, for all $x \in X$. Thus $f(x)=A^{5}(x)$. Conversely, suppose $f(x)=A^{5}(x)$ for all $x \in X$, where $A^{5}(x)$ is the diagonal of the 5 additive symmetric map $A_{5}: X^{5} \rightarrow Y$. We note that

$$
\begin{aligned}
A^{5}(a x+b y)= & a^{5} A^{5}(x)+b^{5} A^{5}(y)+5 a^{4} b A^{4,1}(x, y)+10 a^{3} b^{2} A^{3,2}(x, y) \\
& +10 a^{2} b^{3} A^{2,3}(x, y)+5 a b^{4} A^{1,4}(x, y),
\end{aligned}
$$

for all $x, y \in X$ and $a, b \in \mathbb{Q}$. The remains of the proof can be easily checked.

\section{Stabilities}

Throughout this section, let $X$ be a quasi $\beta$-normed space and let $Y$ be a quasi $\beta$-Banach space with a quasi $\beta$-norm $\|\cdot\|_{Y}$. Let $K$ be the modulus of concavity of $\|\cdot\|_{Y}$. We will investigate the generalized Hyers-Ulam-Rassias stability problem for the functional equation (1.2). For a given mapping $f: X \rightarrow Y$, let

$$
\begin{aligned}
D f(x, y):= & f(3 x+y)+f(3 x-y)+5[f(x+y)+f(x-y)] \\
& -4[f(2 x+y)+f(2 x-y)]-2 f(3 x)+246 f(x),
\end{aligned}
$$

$x, y \in X$.

Theorem 3.1. Suppose that there exists a mapping $\phi: X^{2} \rightarrow \mathbb{R}^{+}:=$ $[0, \infty)$ for which a mapping $f: X \rightarrow Y$ satisfies $f(0)=0$,

$$
\|D f(x, y)\|_{Y} \leq \phi(x, y)
$$

and the series $\sum_{j=0}^{\infty}\left(\frac{K}{32^{\beta}}\right)^{j} \phi\left(2^{j} x, 2^{j} y\right)$ converges for all $x, y \in X$. Then there exists a unique quintic mapping $Q: X \rightarrow Y$ which satisfies the equation (1.2) and the inequality

$$
\|f(x)-Q(x)\|_{Y} \leq \frac{K}{256^{\beta}} \sum_{j=0}^{\infty}\left(\frac{K}{32^{\beta}}\right)^{j} \phi\left(2^{j} x, 0\right),
$$

for all $x \in X$. 
Proof. By letting $y=0$ in the inequality (3.1), we have

$$
\|D f(x, 0)\|_{Y}=\|256 f(x)-8 f(2 x)\|_{Y} \leq \phi(x, 0),
$$

that is,

$$
\left\|f(x)-\frac{1}{32} f(2 x)\right\|_{Y} \leq \frac{1}{256^{\beta}} \phi(x, 0),
$$

for all $x \in X$. For any positive integer $k$,

$$
\left\|\left(\frac{1}{32}\right)^{k} f\left(2^{k} x\right)-\left(\frac{1}{32}\right)^{k+1} f\left(2^{k+1} x\right)\right\|_{Y} \leq \frac{1}{256^{\beta}} \frac{1}{32^{k \beta}} \phi\left(2^{k} x, 0\right),
$$

for all $x \in X$. For any positive integers $n$ and $m$ with $n>m$,

$$
\left\|\left(\frac{1}{32}\right)^{m} f\left(2^{m} x\right)-\left(\frac{1}{32}\right)^{n} f\left(2^{n} x\right)\right\|_{Y} \leq \frac{1}{256^{\beta}} \frac{1}{K^{m-1}} \sum_{j=m}^{n-1}\left(\frac{K}{32^{\beta}}\right)^{j} \phi\left(2^{j} x, 0\right),
$$

for all $x \in X$. As $n \rightarrow \infty$, the right-hand side in the inequality (3.6) close to 0. Hence $\left\{\left(\frac{1}{32}\right)^{n} f\left(2^{n} x\right)\right\}$ is a Cauchy sequence in the quasi $\beta$-Banach space $Y$. Thus we can define a mapping $Q: X \rightarrow Y$ by

$$
Q(x)=\lim _{n \rightarrow \infty}\left(\frac{1}{32}\right)^{n} f\left(2^{n} x\right),
$$

for all $x \in X$.

By letting $m=0$ in the inequality (3.6), we have

$$
\left\|f(x)-\left(\frac{1}{32}\right)^{n} f\left(2^{n} x\right)\right\|_{Y} \leq \frac{K}{256^{\beta}} \sum_{j=0}^{n-1}\left(\frac{K}{32^{\beta}}\right)^{j} \phi\left(2^{j} x, 0\right),
$$

for all $x \in X, n \in \mathbb{N}$. As $n \rightarrow \infty$ in the inequality (3.7),

$$
\|f(x)-Q(x)\|_{Y} \leq \frac{K}{256^{\beta}} \sum_{j=0}^{\infty}\left(\frac{K}{32^{\beta}}\right)^{j} \phi\left(2^{j} x, 0\right),
$$

for all $x \in X$. It satisfies the inequality (3.2). Now, replacing $x$ and $y$ by $2^{n} x$ and $2^{n} y$ respectively and dividing by $32^{\beta n}$ in the inequality (3.1), we have

$$
\left(\frac{1}{32^{\beta}}\right)^{n}\left\|D f\left(2^{n} x, 2^{n} y\right)\right\|_{Y} \leq\left(\frac{K}{32^{\beta}}\right)^{n} \phi\left(2^{n} x, 2^{n} y\right),
$$

for all $x, y \in X$. By taking $n \rightarrow \infty$, the definition of $Q$ implies that $Q$ satisfies (1.2) for all $x, y \in X$, that is, $Q$ is the quintic mapping. Next, it remains to show the uniqueness. Assume that there exists 
$T: X \rightarrow Y$ satisfying (1.2) and (3.2). The Theorem 2.1 implies that $T\left(2^{n} x\right)=2^{5 n} T(x)$ and $Q\left(2^{n} x\right)=2^{5 n} Q(x)$, for all $x \in X$. Then

$$
\begin{aligned}
& \|T(x)-Q(x)\|_{Y} \\
& =\left(\frac{1}{32^{\beta}}\right)^{n}\left\|T\left(2^{n} x\right)-Q\left(2^{n} x\right)\right\|_{Y} \\
& \leq\left(\frac{1}{32^{\beta}}\right)^{n} K\left(\left\|T\left(2^{n} x\right)-f\left(2^{n} x\right)\right\|_{Y}+\left\|f\left(2^{n} x\right)-Q\left(2^{n} x\right)\right\|_{Y}\right) \\
& \leq \frac{2 K^{2}}{256^{\beta}} \sum_{j=0}^{\infty}\left(\frac{K}{32^{\beta}}\right)^{n+j} \phi\left(2^{n+j} x, 0\right),
\end{aligned}
$$

for all $x \in X$. By letting $n \rightarrow \infty$, we immediately have the uniqueness of $Q$.

Theorem 3.2. Suppose that there exists a mapping $\phi: X^{2} \rightarrow \mathbb{R}^{+}:=$ $[0, \infty)$ for which a mapping $f: X \rightarrow Y$ satisfies

$$
\|D f(x, y)\|_{Y} \leq \phi(x, y)
$$

and the series $\sum_{j=1}^{\infty}\left(32^{\beta} K\right)^{j} \phi\left(2^{-j} x, 2^{-j} y\right)$ converges for all $x, y \in X$. Then there exists a unique quintic mapping $Q: X \rightarrow Y$ which satisfies the equation (1.2) and the inequality

$$
\|f(x)-Q(x)\|_{Y} \leq \frac{1}{8^{\beta}} \sum_{j=1}^{\infty}\left(32^{\beta} K\right)^{j} \phi\left(2^{-j} x, 0\right),
$$

for all $x \in X$.

Proof. If $x$ is replaced by $\frac{1}{2} x$ in the inequality (3.3), then the proof follows from the proof of Theorem 3.1.

Now, we will investigate the stability of the given qurtic functional equation (1.2) using the alternative fixed point method. Before proceeding the proof, we will state the theorem, the alternative of fixed point.

Definition 3.3. Let $X$ be a set. A function $d: X \times X \rightarrow[0, \infty]$ is called a generalized metric on $X$ if $d$ satisfies

(1) $d(x, y)=0$ if and only if $x=y$;

(2) $d(x, y)=d(y, x)$ for all $x, y \in X$;

(3) $d(x, z) \leq d(x, y)+d(y, z)$ for all $x, y, z \in X$.

Theorem 3.4 ( The alternative of fixed point [13], [22] ). Suppose that we are given a complete generalized metric space $(\Omega, d)$ and a 
strictly contractive mapping $T: \Omega \rightarrow \Omega$ with Lipschitz constant $L$. Then for each given $x \in \Omega$, either

$$
d\left(T^{n} x, T^{n+1} x\right)=\infty \text { for all } n \geq 0,
$$

or there exists a natural number $n_{0}$ such that

1. $d\left(T^{n} x, T^{n+1} x\right)<\infty$ for all $n \geq n_{0}$;

2. The sequence $\left(T^{n} x\right)$ is convergent to a fixed point $y^{*}$ of $T$;

3. $y^{*}$ is the unique fixed point of $T$ in the set

$$
\triangle=\left\{y \in \Omega \mid d\left(T^{n_{0}} x, y\right)<\infty\right\} ;
$$

4. $d\left(y, y^{*}\right) \leq \frac{1}{1-L} d(y, T y)$ for all $y \in \triangle$.

Theorem 3.5. Let $f: X \rightarrow Y$ be a function with $f(0)=0$ for which there exists a function $\phi: X^{2} \rightarrow[0, \infty)$ such that there exists a constant $L, 0<L<1$, satisfying the inequalities

$$
\begin{gathered}
\|D f(x, y)\|_{Y} \leq \phi(x, y) \\
\phi(2 x, 2 y) \leq 32^{\beta} L \phi(x, y),
\end{gathered}
$$

for all $x, y \in X$. Then there exists a unique quintic function $Q: X \rightarrow Y$ defined by $\lim _{n \rightarrow \infty} \frac{f\left(2^{n} x\right)}{32^{n}}=Q(x)$ such that

$$
\|f(x)-Q(x)\|_{Y} \leq \frac{1}{256^{\beta}(1-L)} \phi(x, 0),
$$

for all $x \in X$.

Proof. Consider the set

$$
\Omega=\{g \mid g: X \rightarrow Y, g(0)=0\}
$$

and introduce the generalized metric on $\Omega$,

$$
d(g, h)=\inf \left\{\mu \in(0, \infty) \mid\|g(x)-h(x)\|_{Y} \leq \mu \phi(x, 0), \text { for all } x \in X\right\} .
$$

It is easy to show that $(\Omega, d)$ is complete. Now we define a function $T: \Omega \rightarrow \Omega$ by

$$
T g(x)=\frac{1}{32} g(2 x), \quad g \in \Omega
$$

for all $x \in X$. Note that for all $g, h \in \Omega$, let $\mu \in(0, \infty)$ be an arbitrary constant with $d(g, h) \leq \mu$. Then 


$$
\begin{aligned}
& \|g(x)-h(x)\|_{Y} \leq \mu \phi(x, 0), \text { for all } x \in X, \\
\Rightarrow & \left\|\frac{1}{32} g(2 x)-\frac{1}{32} h(2 x)\right\|_{Y} \leq \frac{1}{32^{\beta}} \mu \phi(2 x, 0), \text { for all } x \in X, \\
\Rightarrow & \left\|\frac{1}{32} g(2 x)-\frac{1}{32} h(2 x)\right\|_{Y} \leq L \mu \phi(x, 0), \text { for all } x \in X, \\
\Rightarrow & d(T g, T h) \leq L \mu .
\end{aligned}
$$

Hence we have that

$$
d(T g, T h) \leq L d(g, h),
$$

for all $g, h \in \Omega$, that is, $T$ is a strictly self-mapping of $\Omega$ with the Lipschitz constant $L$. By setting $y=0$ in the inequality (3.11) and dividing both sides by $256^{\beta}$, then we have

$$
\left\|f(x)-\frac{1}{32} f(2 x)\right\|_{Y} \leq \frac{1}{256^{\beta}} \phi(x, 0),
$$

for all $x \in X$, that is, $d(T f, f) \leq \frac{1}{256^{\beta}}<\infty$. We can apply the fixed point alternative and since $\lim _{r \rightarrow \infty} d\left(T^{r} f, Q\right)=0$, there exists a fixed point $Q$ of $T$ in $\Omega$ such that

$$
Q(x)=\lim _{n \rightarrow \infty} \frac{f(2 x)}{32^{n}},
$$

for all $x \in X$. Letting $x=2^{n} x$ and $y=2^{n} y$ in the equation (3.11) and dividing by $2^{3 n \beta}$,

$$
\begin{aligned}
\|D Q(x, y)\|_{Y} & =\lim _{n \rightarrow \infty} \frac{\left\|D f\left(2^{n} x, 2^{n} y\right)\right\|_{Y}}{32^{n \beta}} \\
& \leq \lim _{n \rightarrow \infty} \frac{1}{32^{n \beta}} \phi\left(2^{n} x, 2^{n} y\right) \\
& \leq \lim _{n \rightarrow \infty} L^{n} \phi(x, y)=0,
\end{aligned}
$$

for all $x, y \in X$; that is it satisfies the equation (1.2). Hence the $Q$ is quintic. Also, the fixed point alternative guarantees that such a $Q$ is the unique function. Again using the fixed point alternative, we have

$$
d(f, Q) \leq \frac{1}{1-L} d(T f, f) .
$$

Hence we may conclude that

$$
d(f, Q) \leq \frac{1}{1-L} d(T f, f) \leq \frac{1}{256^{\beta}(1-L)},
$$

which implies the equation (3.12). 
Theorem 3.6. Let $f: X \rightarrow Y$ be a function with $f(0)=0$ for which there exists a function $\phi: X^{2} \rightarrow[0, \infty)$ such that there exists a constant $L, 0<L<1$, satisfying the inequalities

$$
\begin{gathered}
\|D f(x, y)\|_{Y} \leq \phi(x, y) \\
\phi(x, y) \leq \frac{L}{32^{\beta}} \phi(2 x, 2 y),
\end{gathered}
$$

for all $x, y \in X$. Then there exists a unique quintic function $Q: X \rightarrow Y$ defined by $\lim _{n \rightarrow \infty} 32^{n} f\left(\frac{1}{2^{n}} x\right)=Q(x)$ such that

$$
\|f(x)-Q(x)\|_{Y} \leq \frac{L}{256^{\beta}(1-L)} \phi(x, 0),
$$

for all $x \in X$.

Proof. We will use the same notation for $\Omega$ and $d$ as in the proof of Theorem 3.5 and then we define a function $T: \Omega \rightarrow \Omega$ by

$$
T g(x)=32 g\left(\frac{x}{2}\right), \quad g \in \Omega
$$

for all $x \in X$. Then

$$
\begin{aligned}
\|T g(x)-T h(x)\|_{Y} & =32^{\beta}\left\|g\left(\frac{x}{2}\right)-h\left(\frac{x}{2}\right)\right\|_{Y} \\
& \leq 32^{\beta} K \phi\left(\frac{x}{2}, 0\right) \leq L K \phi(x, 0),
\end{aligned}
$$

for all $x \in X$, that is, $d(T g, T h) \leq L K$. Hence $d(T g, T h) \leq L d(g, h)$, for any $g, h \in \Omega$. Thus $T$ is a strictly self-mapping of $\Omega$ with the Lipschitz constant $L$. By letting $x=\frac{x}{2}$ and $y=0$ in the inequality (3.14) and dividing both sides by $8^{\beta}$, then

$$
\left\|f(x)-32 f\left(\frac{x}{2}\right)\right\|_{Y} \leq \frac{1}{8^{\beta}} \phi\left(\frac{x}{2}, 0\right) \leq \frac{L}{256^{\beta}} \phi(x, 0),
$$

for all $x \in X$. Hence $d(T f, f) \leq \frac{L}{256^{\beta}}<\infty$. The remains follows from the proof of Theorem 3.5.

\section{References}

[1] T. Aoki, On the stability of the linear transformation in Banach spaces, J. Math. Soc. Japan 2 (1950), 64-66.

[2] J. H. Bae and W. G. Park, On the generalized Hyers-Ulam-Rassias stability in Banach modules over a $C^{*}$-algebra, J. Math. Anal. Appl. 294 (2004), 196-205.

[3] Y. Benyamini and J. Lindenstrauss, Geometric Nonlinear Functional Analysis, vol. 1, Colloq. Publ. vol.48, Amer. Math. Soc., Providence, (2000). 
[4] I. G. Cho, D. Kang, and H. Koh, Stability Problems of Quintic Mappings in Quasi- $\beta$-Normed Spaces, Journal of Inequalities and Applications, Article ID 368981, 9 pages, 2010 (2010).

[5] S. Czerwik, On the stability of the quadratic mapping in normed spaces, Abh. Math. Sem. Univ. Hamburg 62 (1992), 59-64.

[6] S. Cherwik, Functional Equations and Inequalities in Several Variables, World Scientific Publ.Co., New Jersey, London, Singapore, Hong Kong, (2002).

[7] Z. Gajda, On the stability of additive mappings, Internat. J. Math. Math. Sci. 14 (1991), 431-434.

[8] M. E. Gordji, Y. J. Cho, M. B. Ghaemi, and H. Majani, Approximately quintic and sextic mappings formn r-divisible groups into into Serstnev Probabilistic Banach Spaces: Fixed Point Method, Disc. Dynam. in Natural and society, Article ID 572062, 16 pages, 2011 (2011).

[9] D. H. Hyers, On the stability of the linear equation, Proc. Nat. Acad. Sci. U.S.A. 27 (1941), 222-224.

[10] D. H. Hyers and Th. M. Rassias, Approximate homomorphisms, Aequationes Mathematicae, 44 (1992), 125-153.

[11] D. H. Hyers, G. Isac, and Th. M. Rassias, Stability of Functional Equations in Several Variables, Birkhauser, Boston, Basel, Berlin, 1998.

[12] S. M. Jung, Hyers-Ulam-Rassias Stability of Functional Equations in Mathematical Analysis, Hadronic Press,Inc., Florida, 2001.

[13] B. Margolis and J. B. Diaz, A fixed point theorem of the alternative for contractions on a generalized complete metric space, Bull. Amer. Math. Soc. 74 (1968), 305-309.

[14] C. Park, J. L. Cui, and M. E. Gordji, Orthogonality and quintic functional equations, Acta Mathematica Sinica, English Series, 29 (2013), no. 7, 13811390.

[15] J. M. Rassias and H. M. Kim Generalized Hyers. Ulam stability for general additive functional equations in quasi- $\beta$-normed spaces, J. Math. Anal. Appl. 356 (2009), 302-309.

[16] Th. M. Rassias, On the stability of the linear mapping in Banach spaces, Proc. Amer. Math. Soc. 72 (1978), 297-300.

[17] Th. M. Rassias, On the stability of functional equations and a problem of Ulam, Acta Applicandae Mathematicae, 62 (2000), 23-130.

[18] Th. M. Rassias, On the stability of functional equations in Banach spaces, J. Math. Anal. Appl. 251 (2000), 264-284.

[19] Th. M. Rassias and P. Šemrl On the Hyers-Ulam stability of linear mappings, J. Math. Anal. Appl. 173 (1993), 325-338.

[20] Th. M. Rassias and K. Shibata, Variational problem of some quadratic functions in complex analysis, J. Math. Anal. Appl. 228 (1998), 234-253.

[21] S. Rolewicz, Metric Linear Spaces, Reidel/PWN-Polish Sci. Publ., Dordrecht, 1984.

[22] I. A. Rus, Principles and Appications of Fixed Point Theory, Ed. Dacia, ClujNapoca, 1979 (in Romanian).

[23] S. M. Ulam, Problems in Morden Mathematics, Wiley, New York, 1960. 
[24] T. Z. Xu, J. M. Rassias, and W. X. Xu, A generalized mixed quadratic-quartic functional equation, Bull. Malaysian Math. Scien. Soc. 35 (2012), no. 3, 633649 .

Department of Mathematical Education Dankook University

Yongin 448-701, Republic of Korea

E-mail: khjmath@dankook.ac.kr 